\title{
MARCAS EM FÓSSEIS DE MEGAFAUNA EM LAGOA DA PEDRA, SALGUEIRO-PE
}

\section{BRANDS ON MEGAFAUNA FOSSILS IN LAGOA DA PEDRA, SALGUEIRO-PE, BRAZIL}

Eduarda Tavares Peters ${ }^{1}$

dudah.peters@yahoo.com.br

Edison Vicente Oliveira ${ }^{2}$



vicenteedi@gmail.com

\section{RESUMO}

Este trabalho descreve marcas encontradas em fósseis de mamíferos pleistocênicos do Sítio Lagoa da Pedra, que fica localizado no distrito de Conceição das Creoulas, identificando possíveis relações temporais e/ou físicas entre os grupos pré-históricos e a megafauna que coabitou a região nordeste do Brasil, em Salgueiro - PE, no Pleistoceno Final - Holoceno Inicial. A metodologia aplicada, utilizando microscópio estereoscópico, identificou fósseis que apresentam marcas que foram possivelmente causadas por instrumentos líticos. O trabalho apresenta indícios de interação homem-megafauna através da caça desses animais, reforçando evidências já existentes sobre a coabitação durante o final do Pleistoceno e início do Holoceno.

Palabras clave: Quaternário, marcas, fósseis, mamíferos pleistocênicos, megamamíferos.

${ }^{1}$ Discente, Programa de Pós-graduação em Arqueologia, UFPE.

${ }^{2}$ Discente, Programa de Pós-graduação em Arqueologia, UFPE.

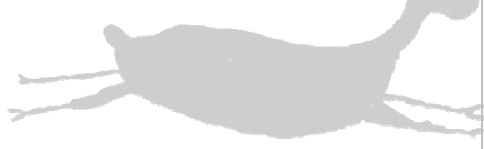

Indexadores: Latindex, ISIS, Google Academic 


\begin{abstract}
This work describes marks found on fossil mammals of the Pleistocene Lagoa da Pedra Site, which is located in the Conceição das Creoulas district, identifying possible temporal and / or physical relationships between prehistoric groups and the megafauna that cohabitated the northeast region of Brazil., in Salgueiro - PE, in the Late Pleistocene - Early Holocene. The applied methodology, using stereoscopic microscope, identified fossils presenting marks that were possibly caused by lithic instruments. This work presents evidence of human-megafauna interaction through hunting of these animals, reinforcing existing evidence on cohabitation during the late Pleistocene and early Holocene.
\end{abstract}

Keywords: Quaternary, marks, fossils, pleistocene mammals, megamammals.

\title{
CONTEXTO DA PESQUISA
}

$\mathrm{Na}$ América do Sul, apesar de haver várias evidências de que os grupos préhistóricos coexistiram com pelo menos algumas das espécies da fauna pleistocênica extinta (Barnosky e Lindsey, 2010; Hubbe et al., 2007, 2009), evidências de caça ainda são poucas (Borrero, 2009).

Um dos vestígios mais antigos (29.050 +- 290 anos AP) de caça de megafauna através de marcas de descarne na América do Sul foi descoberto no Uruguai, sítio Arroyo Vizcaíno (Arribas et al., 2001; Fariña \& Castillo, 2007). No Brasil, há uma série de evidências consistentes de interação, como por exemplo, marcas de descarne em úmero de preguiça (Mylodonopsis ibseni), reportado para a Bahia (Prous, 1992), além de osteodermos utilizados para a elaboração de adornos, como evidenciados no Mato Grosso (Neves \& Piló, 2008). Na região de Lagoa Santa, as cavernas têm oferecido também sinais de evidência de interação. Peter 
Lund conduziu escavações paleontológicas na Gruta do Sumidouro onde encontrou vestígios de convivência entre a megafauna e caçadores coletores préhistóricos (Neves \& Piló, 2003). No abrigo Santa Elina, situado no município de Jangada-MT, foi evidenciado apenas um osso dérmico com marcas antropogênicas (Vialou \& Vialou, 2008). Na caverna Lapa do Caetano, em Minas Gerais foi achado um crânio de um Notiomastodon platensis juvenil com uma perfuração causada por um instrumento pontiagudo (Mothé et al., 2014; AraújoJúnior et al., 2018).

No nordeste do Brasil, fósseis de megafauna e artefatos líticos são encontrados em depósitos sedimentares, do Pleistoceno-Holoceno, de cavernas, depósitos de tanques e abrigos-sob-rocha, principalmente no Parque Nacional da Serra da Capivara (Guidón, 2003), como por exemplo, o sitio Toca da Barra da Janela de Antonião, Sítio do Meio, Boqueirão da Pedra Furada, sítio Toca do João do Leite, sítio Perna I (Mello, 2004; Bélo, 2012; Lucas, 2014; Bélo 2017).

Em depósitos de tanques (incluindo lagoas) o registro paleontológico/arqueológico é muito raro. No Sítio Poço Redondo, Sergipe, registrou-se um fragmento molariforme de Eremotherium com possíveis modificações antrópicas (Dantas et al., 2012; porém ver contestação de Hubbe et al. (2012). No Sítio Uri de Cima, Pernambuco, foi identificado fósseis de Eremotherium e material lítico, sem contudo, apresentar uma correlação espacial entre ambos (Mutzenberg e Valli, 2016). Outros registros de instrumentos líticos 
em tanques são reportados para os distritos de Conceição das Creoulas e Fazenda Nova, Estado de Pernambuco (Bélo, 2017; Galindo et al., 1994; Lima, 1995). O histórico desses achados remonta ao ano de 1993 no Sítio Lagoa da Pedra, em Conceição das Creoulas. Muitos vestígios da presença humana foram registrados no sítio Lagoa da Pedra, incluindo instrumentos líticos, núcleos, seixos com marca de percussão, lascas de vários tamanhos e lâminas de machados polidos (Lima e Viana, 1993; De Paula, 1998). Ao redor da área onde foi encontrado o material referente a esse estudo, há um aglomerado de depressões que formam o Complexo Lacustre das Caraíbas, onde também há ocorrência de fósseis pleistocênicos associados a instrumentos produzidos por grupos pré-histórico (Galindo et al., 1994; Lima, 1995). Os líticos coletados em Fazenda Nova foram descritos por Bélo (2017).

Apesar desses registros paleontológicos/arqueológicos, ainda são poucos os estudos sobre marcas em elementos esqueletais (Rosa et al., 2018). Nesse sentido, os principais trabalhos para o Nordeste brasileiro até o momento são de Dantas et al. (2012) e Bélo \& Oliveira (2013). Os fósseis de Hippidion (Perissodactyla, Equidae) com marcas antropogênicas identificados no Sítio Toca da Janela da Barra do Antonião, sudeste do Piauí (Bélo \& Oliveira, 2013), sugerem que houve atividade de descarne, desarticulação e remoção da medula.

Esse registro em vários municípios do nordeste brasileiro sugere uma ampla ocupação de caçadores coletores já no final do Pleistoceno, um padrão consistente 
com o restante do registro sul-americano (Prous, 1992; Barnosky \& Lindsey, 2010). Os sítios estudados, principalmente aqueles do tipo depósitos de tanques (vide Waldherr et al., 2017 para definição), serviram no passado de reservatórios naturais de água, e muito provavelmente poderiam ter sido usados como território propício para captura de recursos necessários para a manutenção sócio-econômica das comunidades pré-históricas que habitavam aquela região (Santos, 2009).

O presente trabalho propõe analisar e descrever marcas encontradas em fósseis de megamamíferos, encontrados no Sítio Lagoa da Pedra caracterizando possíveis relações temporais ou físicas existentes entre as comunidades caçadoras coletoras e a mastofauna que habitava a região de Salgueiro.

\section{LOCALIZAÇÃO GEOGRÁFICA}

O distrito de Conceição das Creoulas está situado no município de Salgueiro (Fig. 1), no sertão pernambucano, a $518 \mathrm{~km}$ de distância da capital do Recife. Distando $1.1 \mathrm{~km}$ ao norte de Conceição das Creoulas, localiza-se um complexo de três lagoas (Lima, 1995). A maior e de suma importância para a população local é a Lagoa das Caraíbas, ao lado se encontra a Lagoa Vitorino e Lagoa da Pedra, local onde tem sido usado como reservatório de águas pluviais durante o Quaternário (Sanzzoni, 1999), e de onde provém o material usado na pesquisa. 


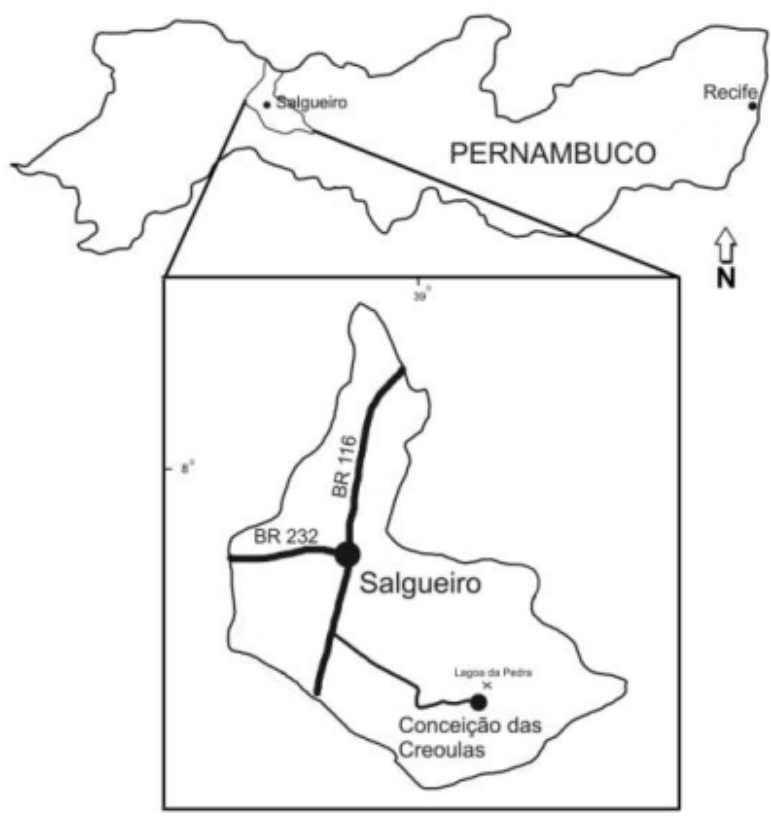

Figura 1. Mapa de localização geográfica. Fonte: Pessoa et al. (2012).

\section{MATERIAL E MÉTODOS}

A metodologia no estudo das marcas consistiu em identificar através de um estudo minucioso a procura de micro-sinais, utilizando-se um microscópio esteroscópio Discovery V.5. Inspecionaram-se possíveis marcas de uso, ou seja, incisões causadas por instrumentos cortantes (ver artigos Binford 1981; Potts \& Shipman, 1981; Shipman \& Rose 1983a; Cook, 1986; Rosa et al., 2018).

Também foi identificada uma fratura em formato longitudinal, determinado segundo os tipos de fratura classificadas por Lyman (1982). 
Aqui será utilizado como referência para a análise das marcas os conceitos propostos por Binford (1981).

O material analisado se encontra armazenado no Laboratório de Paleontologia (Paleolab) sob a sigla DGEO-CTG-UFPE e no Laboratório de Arqueologia (Labifor), ambos da Universidade Federal de Pernambuco (UFPE). Foi analisado um total de 318 fósseis, dos quais, quatro deles foram selecionados para o estudo.

\section{DESCRIÇÃO E DISCUSSÃO}

De todos os fósseis analisados, quatro deles foram identificados com possíveis marcas de corte.

DGEO-CTG-UFPEO 7470 constitui um fragmento proximal de costela provavelmente de Eremotherium laurillardi (Fig. 2A). O mesmo mede $22 \mathrm{~cm}$ de comprimento e 5,5 cm de largura. As marcas apresentam-se na face interna do osso, sendo aproximadamente cinco as identificáveis. As marcas variam de 2,5 a $3,0 \mathrm{~cm}$ de comprimento, e de 3,5 a $3,7 \mathrm{~mm}$ de largura. Apresenta uma incisão alongada com estrias paralelas no sulco, que constitui uma importante característica para identificar a presença de ação antrópica. Uma análise microscópica dessas marcas evidenciou a presença de sulcos paralelos dentro do sulco principal (Fig. 2B). A presença de bordas e a marca possuir a mesma pigmentação do fóssil sugere que ela foi produzida em um momento prédeposicional, ou seja, que o osso ainda estava fresco quando sofreu o golpe (Bélo, 2012). 


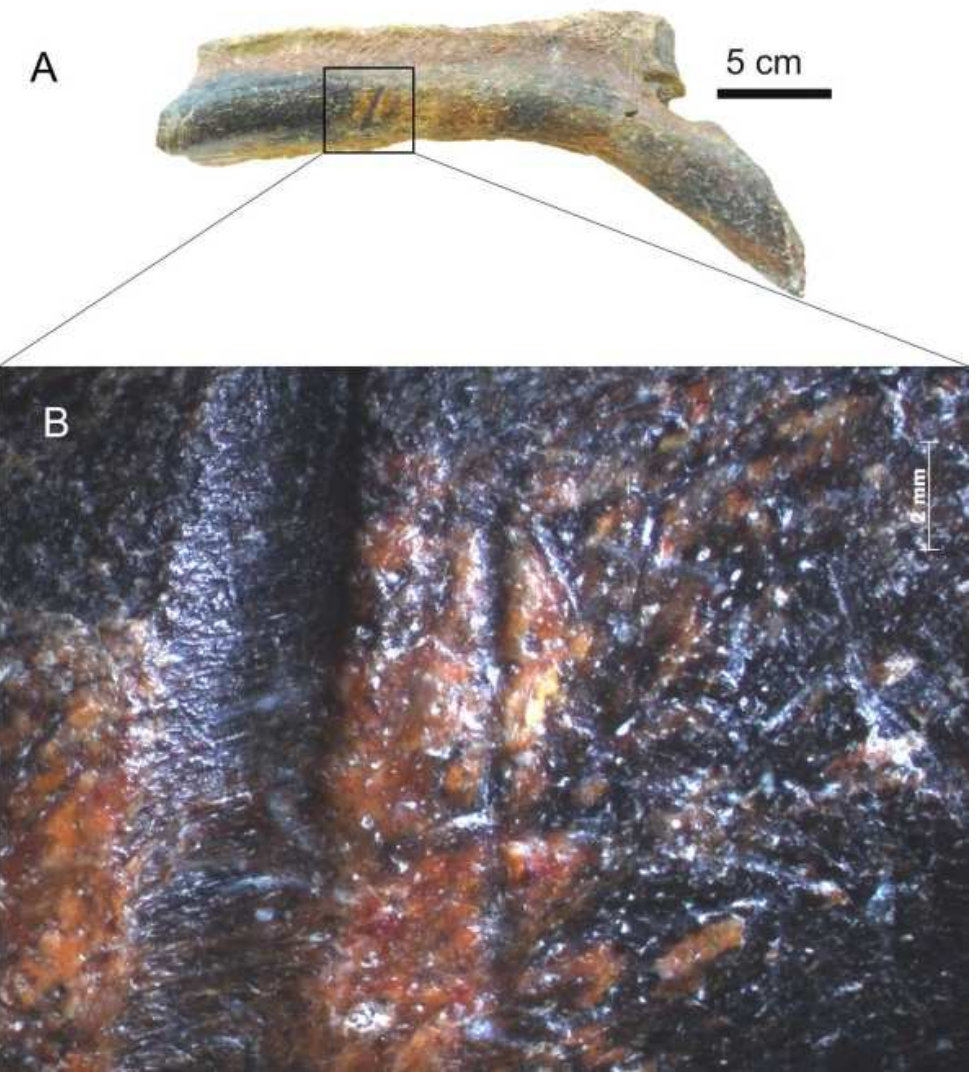

Figura 2

Fragmento de costela de preguiça-gigante de Eremotherium laurillardi (DGEO-CTG-

UFPE 7440) com possível marca antrópica; A- vista geral do elemento ósseo; B- marca fotografada em Microscópico Esteroscópico.

Este fóssil constitui-se em um fragmento não identificado (Fig. 3), de táxon indeterminado e sem numeração, medindo $6 \mathrm{~cm}$ de comprimento e 4,2 $\mathrm{cm}$ de largura. Possui uma incisão que atravessa sua extensão, apresentando $4,2 \mathrm{~cm}$ de comprimento e $0,1 \mathrm{~cm}$ de largura. Após uma análise microscópica foi possível reconhecer sulcos paralelos, dentro do sulco principal (Fig. 3B). 

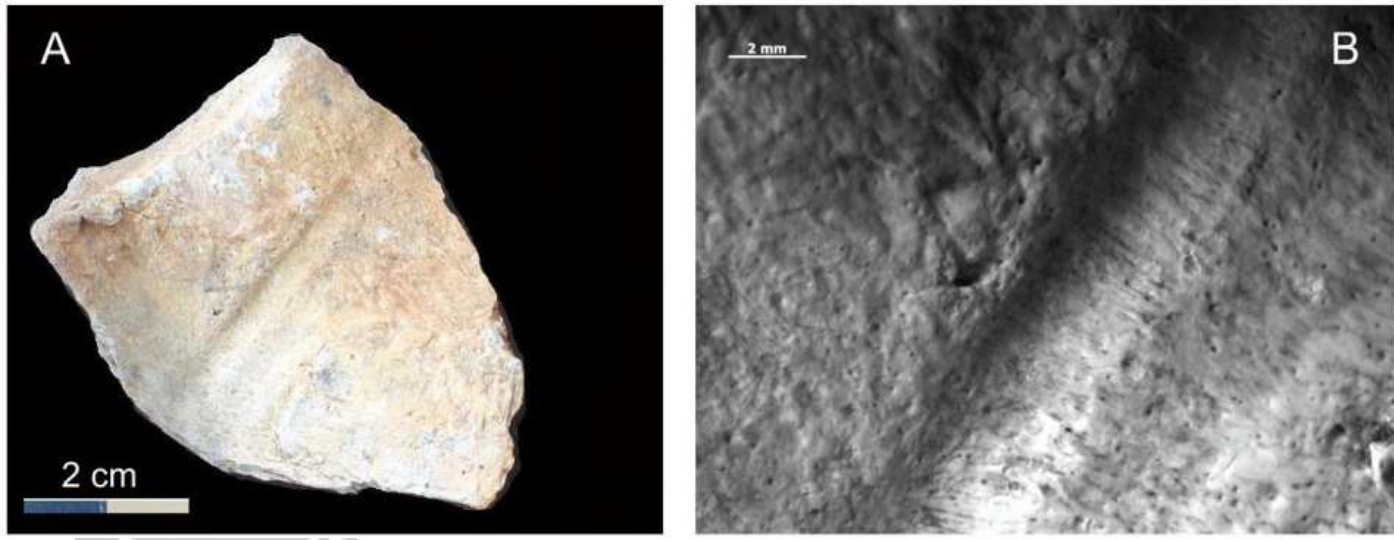

Figura 3. A-fragmento de táxon indeterminado (Labifor s/n); B-marca fotografada em microscópio estereoscópico.

LP-3-8. Esse fóssil (Fig. 4) constitui um fragmento de porção proximal de um metapodial de um cervídeo, medindo $9 \mathrm{~cm}$ de comprimento e $4,8 \mathrm{~cm}$ de largura. Apresenta fraturas de bordas retas e agudas, fazendo com que o osso esteja seccionado longitudinalmente. A porção interna portadora da medula óssea se encontra completamente exposta. Um padrão de fratura semelhante foi descrito por Bélo (2012), caracterizando o método de descarne, com feições de faturamento longitudinal, com ausência de bordas polidas, arredondadas ou abrasão. 


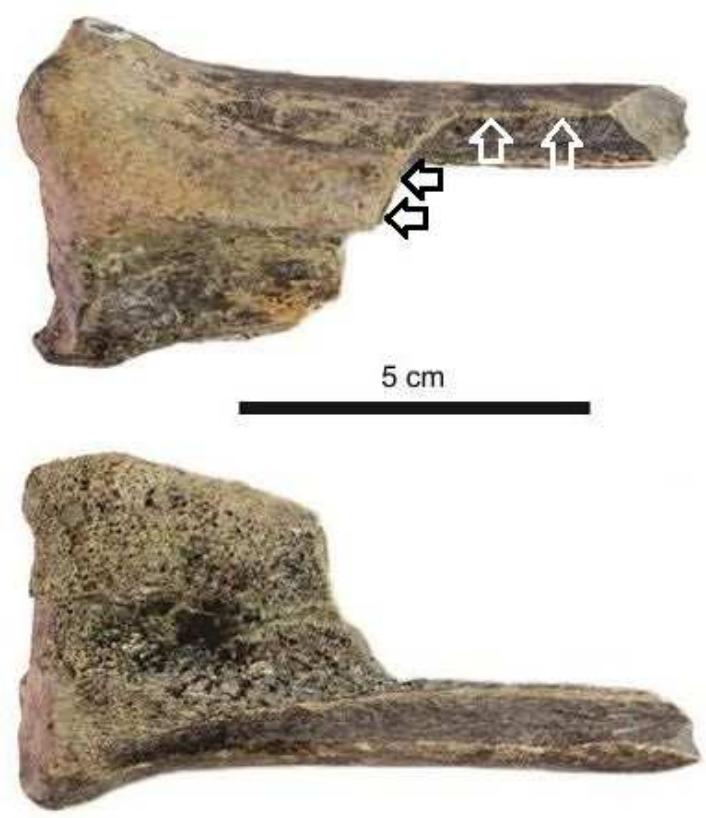

Figura 4. Porção proximal de metapodial, com marca longitudinal (Labifor LP3-20).

DGEO-CTG-UFPE 8742. Este fóssil trata-se de uma costela esternal esquerda de Eremotherium laurillardi (Fig. 5A) medindo 42,1 cm de comprimento, 9,8 cm de largura proximal e 10,6 cm de largura lateral. Possui 2 marcas variando de $1 \mathrm{~cm}$ a $1,5 \mathrm{~cm}$ de comprimento e $0,1 \mathrm{~cm}$ a $0,2 \mathrm{~cm}$ de largura. A figura $5 \mathrm{~B}$ mostra detalhes da região onde se apresenta as marcas e a figura 5C apresenta a morfologia da marca fotografada em microscópio estereoscópico. 

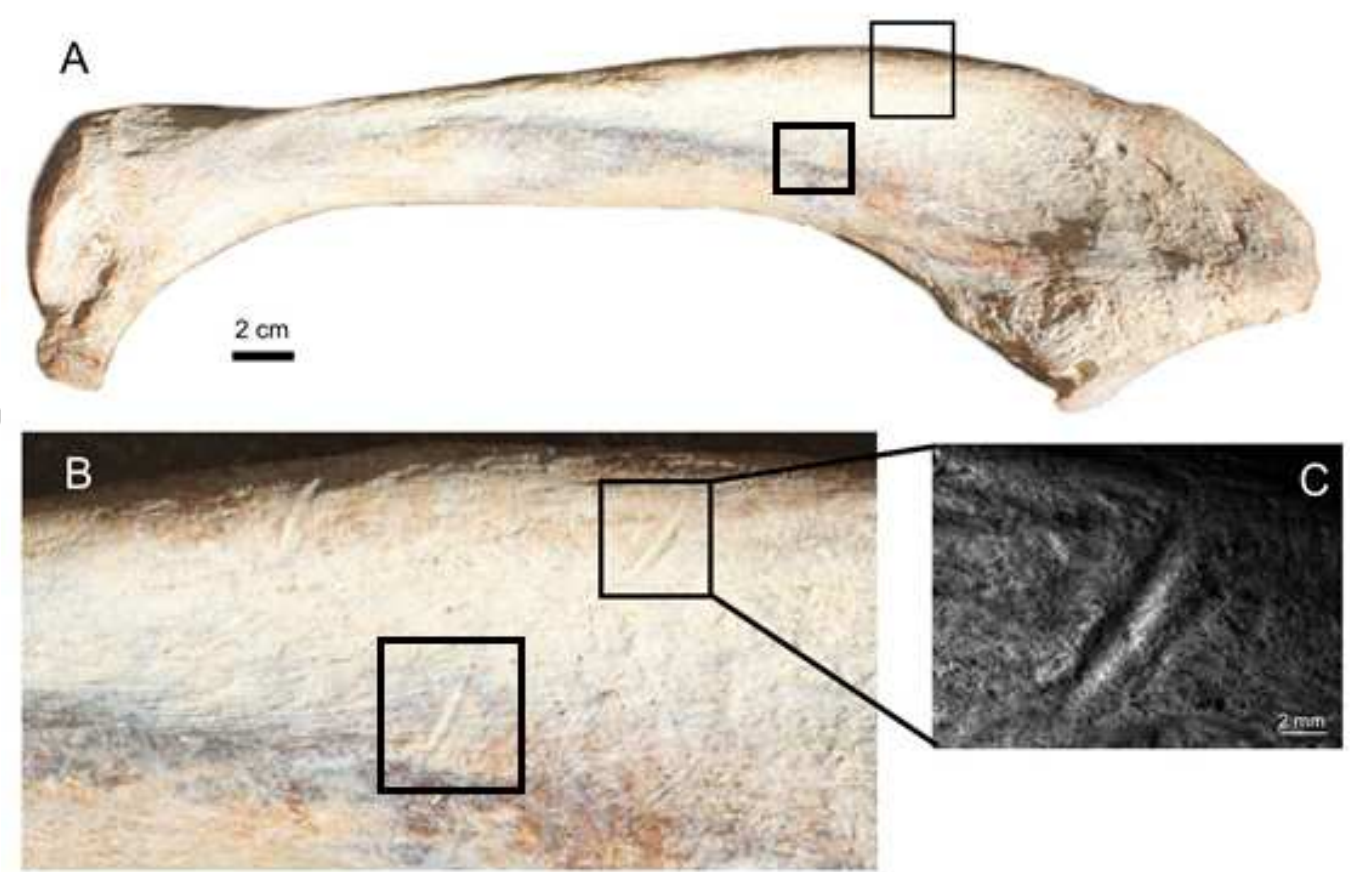

Figura 5. A- Costela esternal esquerda de Eremotherium laurillardi (DGEO-CTG-UFPE 8742); B- Detalhes da região exibindo marcas; C- Morfologia da marca fotografada em microscópio estereoscópico.

A identificação de possíveis evidências de interação antrópicas com a megafauna no material analisado foi, provavelmente, originária de um comportamento de caça através da utilização de instrumentos cortantes. Os vestígios achados no território brasileiro são em sua maioria encontrados em ossos de mamíferos de médio porte (Rosa et al., 2018), com raras exceções (Dantas et al., 2012). Contudo, há de se ressaltar que existem poucos registros documentados sobre 
essas interações, levando em consideração a grande ocorrência de fósseis pleistocênicos por todas as regiões brasileiras. A ausência de mais fósseis com esse tipo de marca talvez se dê em razão da predação dessas espécies terem sido em menor grau e de forma oportunista (Arribas et al., 2001; Borrero \& Martin, 2012; Hubbe et al., 2013; Grayson \& Meltzer, 2015; Araújo-Júnior, 2018).

Os achados registrados no presente trabalho a partir evidências oriundas de sítios escavados, com controle estratigráfico e tafonômico, apontam para o fato de haver fortes evidências de uma interação entre caçadores coletores e a megafauna no final do Pleistoceno e início do Holoceno no Nordeste do Brasil, em especial aquelas do tanque Sítio Lagoa da Pedra. Nas evidências ósseas estudadas, como exemplificadas pelo tipo de fratura longitudinal em um fragmento metapodial na porção proximal de um cervídeo (Fig. 4), repete-se um padrão similar ao que foi encontrado em osso de Hippidion no Sitio Toca da janela da Barra do Antonião, datado do início do Holoceno (Bélo, 2012). Também foi reportado um tipo de incisão alongada, larga e com estrias paralelas em seu interior, esse mesmo tipo de marca também foi evidenciado no sítio TJBA. Contudo, uma diferença importante pode ser vista nesses casos, é que duas das amostras ósseas aqui estudadas (Figs. 2-5) configuram espécimes de megamamíferos pesando acima de uma tonelada (Eremotherium laurillardi), enquanto que as evidências de interação da Toca da Janela da Barra do Antonião se registram em mamíferos de médio porte, pesando menos de 1 tonelada, como o equídeo Hippidion sp. (Bélo, 2017). 


\section{CONCLUSÕES}

Existem poucos registros documentando a interação entre fósseis de megafauna pleistocênica e restos culturais. Os vestígios de interação evidenciados no território brasileiro são em sua grande maioria elaborados em ossos de mamíferos de médio porte.

No fragmento de costela analisado, bem como no fragmento de táxon indeterminado é possível verificar marcas bem semelhantes, ou seja, uma ranhura longa e grossa, com presença de sulcos paralelos no seu interior, possivelmente causada por ação antrópica.

No fragmento metarcapal porção proximal é possível observar uma marca longitudinal com bordas retas e agudas, e a porção interna, onde se encontra a medula, está completamente exposta, sendo muito provavelmente fruto de ação antrópica. Esse tipo de fratura ocorre com o osso ainda em seu estado fresco, não mineralizado.

Não obstante, posteriores análises poderão revelar novos vestígios antrópicos, reforçando as evidências sobre a coabitação, em razão da quantidade de material coletado na escavação durante a campanha, deixando, portanto, em aberto a possibilidade de novas descobertas. 


\section{REFERÊNCIAS BIBLIOGRÁFICAS}

ALMEIDA, J. A. C; MACARIO, J. A; SCHULTZ, R. R. Diagnóstico paleontológico do jazigo fóssil de Lagoa de Dentro, Puxinanã, Paraíba. In: Reunião da Sociedade Brasileira de Paleontologia. Recife, 67pp, 1999.

ALVES, R. S. Os Mamíferos Pleistocênicos de Fazenda Nova, Brejo da Madre de Deus, Pernambuco: Aspectos tafonômicos, taxonômicos e paleoambientais. Dissertação de Mestrado. Pós-graduação em Geociências - UFPE, 163pp, 2007.

ARAÚJO-JÚNIOR, H. I; DA SILVA, L. H. M., ROSA, G. M. Interações entre Humanos Pré-Históricos e a Megafauna Quaternária na América do Sul: Aspectos Zooarqueológicos e Paleoecológicos. Anuário do Instituto de Geociências - UFRJ. Vol. 41 - 1/p. 296-307, 2018.

ARRIBAS, A; PALMQVIST, P; PERES-CLAROS, J. A; CASTILLA, R., VIZCAÍNO, S. F; FARIÑA, R. A. New evidence on the interaction between humans and megafauna in south America. Publicaciones del Seminario de Paleontologia de Zaragoza 5: 228-238, 2001.

BARNOSKY, A. D. \& LINDSEY, M. L. Timing of Quaternay megafaunal extinction in South America in relation to human arrival and climate change. Quaternary International 217 (1-2): 10-29, 2010.

BÉLO, P. S. Alterações antrópicas em restos fósseis da megafauna: tafonomia do sitio arqueológico e paleontológico "Toca da Janela da Barra do Antonião", área arqueológica do Parque Nacional da Serra da Capivara, Piauí, Brasil. Dissertação de Mestrado. Pósgraduação em Geociências - UFPE, 217pp, 2012.

BÉLO, P. S. Extinção e a interação homem-megafauna no final do Pleistoceno e início do Holoceno, nos estados de Pernambuco e Pauí, Nordeste do Brasil. Tese de Doutorado. Pós-graduação - UFPE, 167pp, 2017.

BINFORD, L. R. Bones: Ancient Men and Modern Myths Academic Press, INC, New York, 320pp, 1981. 
BORREIRO, L. A., MARTIN, F. M. Taphonomic observations on ground sloth bone and dung from Cueva del Milodón, Última Esperanza, Chile: 100 years of research history. Quaternary International, 278: p3-11, 2012.

CASTILlo, P. V. \& SIMÕES-LOPES, P. C. A. Registros de modificação óssea em restos faunísticos arqueológicos de mamíferos marinhos. Canindé 12:173-190, 2008.

DA SILVA, F. M. Tafonomia em Tanque de Fazenda Nova, Município de Brejo da Madre de Deus, Estado de Pernambuco, Nordeste do Brasil. Tese de Doutorado. Pósgraduação em Geociências, 97pp, 2013.

DANTAS, M. A. T. Primeiro registro de fósseis de mamíferos do Pleistoceno final Holoceno em cavernas do Estado de Sergipe, Brasil. Revista Brasileira de Paleontologia, 12(2): 161-164, 2009.

DANTAS, M. A. T. Contribuição ao Conhecimento da Megafauna Pleistocênica da Região Intertropical Brasileira. Tese de Doutorado. Pós-graduação em Ecologia, Conservação e Manejo da vida silvestre - UFMG, 105pp, 2012.

DOS SANTOS, C. A; DA SILVA, D. G., CORRÊA, A. C. B. O uso da Abordagem Geomorfológica como Ferramenta para a Compreensão da Escolha de Áreas de Localização dos Sítios Pré-Históricos em Fazenda Nova, Pernambuco. CLIO Sério Arqueológica n23, pp153-169, 2008.

FARIÑA, R. A. \& CASTILLA, R. Earliest evidence for human-megafauna interaction in the Americas. In: Corona-M E, Arroyo-Cabrales J. (eds.). Human and Faunal Relationships Reviewed: an Archaeozoological Approach BAR S1627: 31-33. ii+121 pp. Oxford, Archaeopress, 2007.

GALINDO, M; VIANA, M. S. S; AGOSTINHO, S. Projeto arqueológico/paleontológico Lagoa das Caraíbas, Salgueiro, PE. Revista de Arqueologia, São Paulo, 8(1): 117-131, 1994.

GRAYSON, D. K. \& MELTZER, D. J. Clovis hunting-revisited. Journal of Archaeological Science, 56: 177-193, 2015. 
HUBBE, A; HUBBE, M. \& NEVES, W. A. The Brazilian megamastofauna of the Pleistocene/Holocene transition and its relationship with the early human settlement of the continent. Quaternary International, 118: 1-10, 2013.

LIMA, M. G., VIANA, M. S. S. Arqueologia em Salgueiro Pernambuco. CLIO Série Arqueológica n9, pp61-67, 1993.

LIMA, M. G. Ocupações Pré-Históricas em Conceição das Creoulas Salgueiro, PE. CLIO - Revista de Pesquisa Histórica. Dissertação de Mestrado, Pós-graduação em História UFPE 89pp, 1995.

LYMAN, R. L. Archaeofaunas and subsistence studies. Advances in Archaeological Method and Theory Vol 5, pp331-393, 1982.

LUCAS, L. O. Mudanças técnicas da transição Pleistoceno-Holoceno ao Holoceno Médio no intnerior do nordeste: indústrias líticas da sequencia arqueologica da Toca João Leite PI. Dissertação de Mestrado, Pós-graduação em Arqueologia - UFPE, 160pp, 2014.

MELlO, P. P. A transição do Pleistoceno ao Holoceno no Parque Nacional Serra da Capivara - Piauí - Brasil: uma contribuição ao estudo sobre a antiguidade da presença humana no sudeste do Pauí. Tese de Doutorado, Pós-graduação em História, UFPE, pp 377, 2004.

NEVES, W. A., PILÓ, L. B. O Povo de Luzia - Em busca dos primeiros americanos. Ed. Globo, São Paulo, 336pp, 2008.

PROUS, A. Arqueologia brasileira. Ed. Universidade de Brasília, Brasília, 613pp, 1992. SANTOS, J. S. Ocupação humana, caatinga, paleoambientes e mudanças ambientais nos sertões nordestinos. João Pessoa: JRC Gráfica e Editora, 93pp, 2009.

SANZZONI, M.. Vestígios Arqueológicos na Formação Cacimbas: Sítio Lagoa da Pedra, Salgueiro / Pernambuco. In: X Reunião Científica da Sociedade de Arqueologia Brasileira, Recife - PE. Arqueologia e Preservação do Meio Ambiente, p. 166-166, 1999.

WALDHERR, F. R; ARAÚJO-JÚNIOR, H. I; RODRIGUES, S. W. O. Origem e morfologia dos tanques naturais do Nordeste do Brasil. Pesquisas em Geociências, 44 (3): 467-488, 2017 